\title{
APPLYING GRAPH THEORY TO PROBLEMS IN AIR TRAFFIC MANAGEMENT
}

Amir Farrahi (Universities Space Research Association)

Alan Goldberg (Kestrel Institute)

Leonard Bagasol (Universities Space Research Association)

Jaewoo Jung (NASA Ames Research Center) 


\section{OUTLINE}

- Introduction and motivation

- Background

- Three ATM Problems

- Airspace sectorization problem

- Minimum delay scheduling in traffic flow management

- Maximum dependent set of an aircraft in arrival scheduling

- Summary

- Conclusion 


\section{INTRODUCTION \& MOTIVATION}

- Use known problems to learn about new problem

- Use graph theoretic problems as a suitable substrate

- Bridging isolated islands of knowledge

- Gaining insights about inherent difficulty of new problems

- Solving new problems efficiently using what is known about related problems

- Reap the benefits of development in other technical domains 


\section{INTRODUCTION \& MOTIVATION}

- Learn about new problems by

- Linking them to known problems

- Polynomial "transformation" or "reduction"

- Three examples from graph theory to ATM

- Airspace sectorization problem (ASP)

- Minimum delay scheduling (MDS)

- Maximum set of dependent aircraft (MSDA)

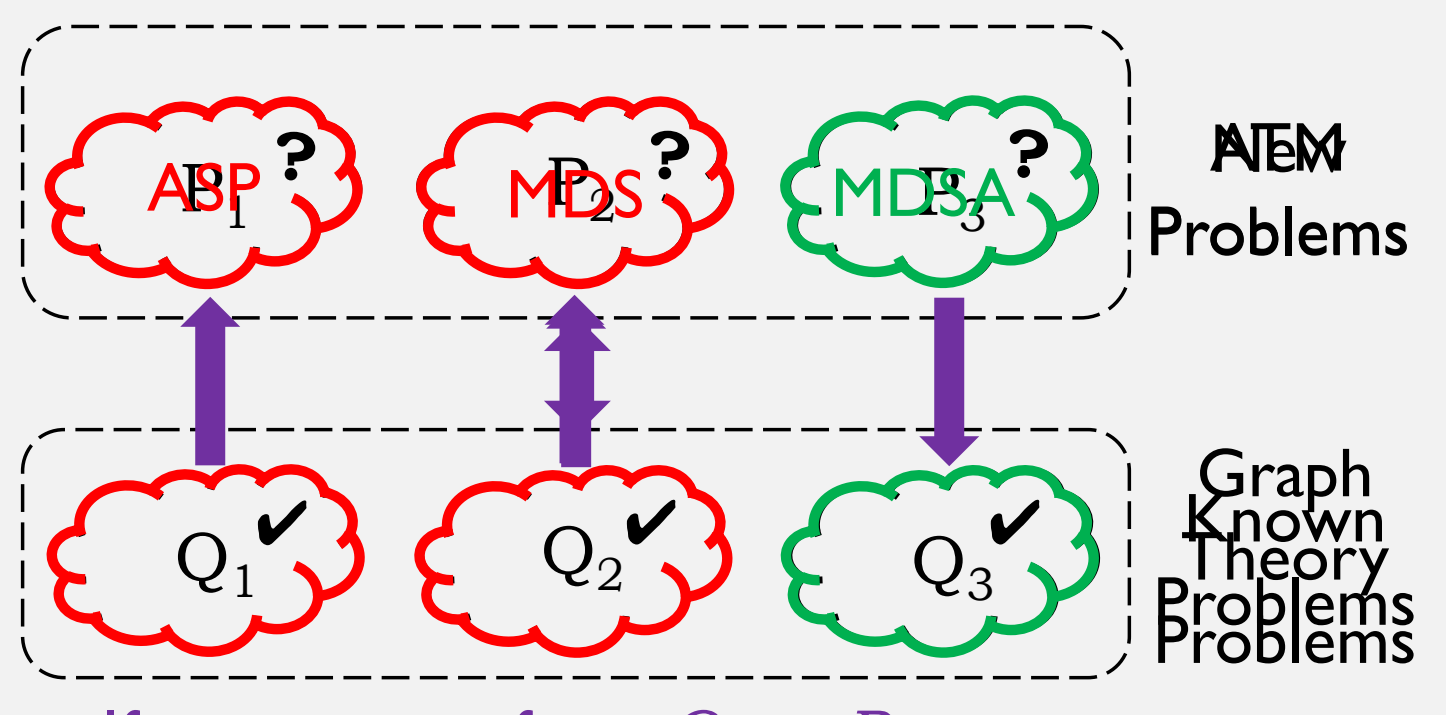

If we can transform $Q_{1}$ to $P_{1}$

And we know $Q_{1}$ is hard to solve

Then $P_{1}$ must be hard to solve

If we can transform $P_{3}$ to $Q_{3}$

And we know how to solve $Q_{3}$

To solve $P_{3}$ : Transform it to $Q_{3}$, then solve 


\section{BACKGROUND: COMPUTATIONAL COMPLEXITY}

- Problems have different inherent difficulty

- Example: Sorting an array of $n$ distinct integers

- One correct solution among $n$ ! permutations

- Know an $\mathrm{O}(\mathrm{n} \log \mathrm{n})$ time algorithm

- Naive algorithm:

- Search among all permutations

- $O(n !)$

Increase in

problem size

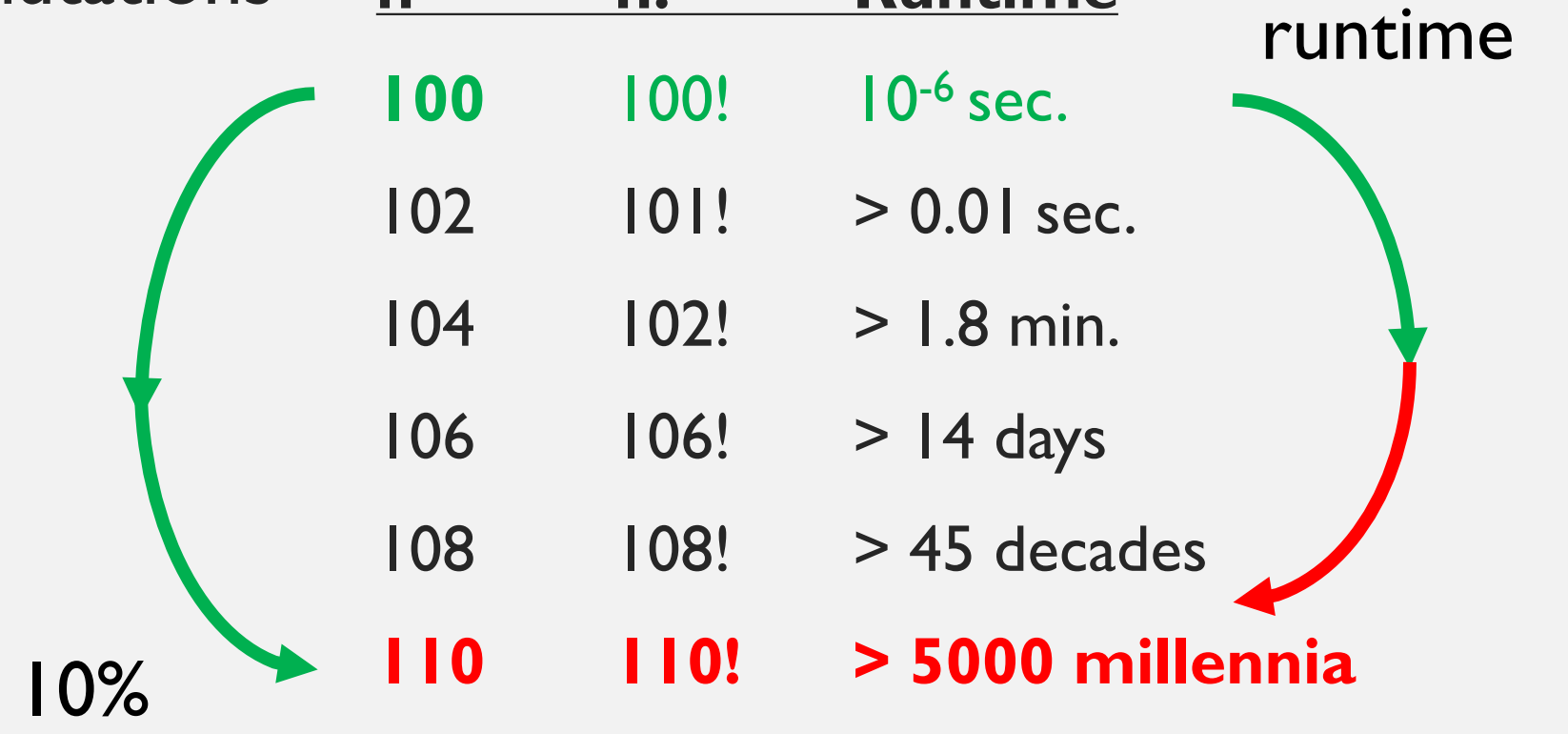

Ridiculously

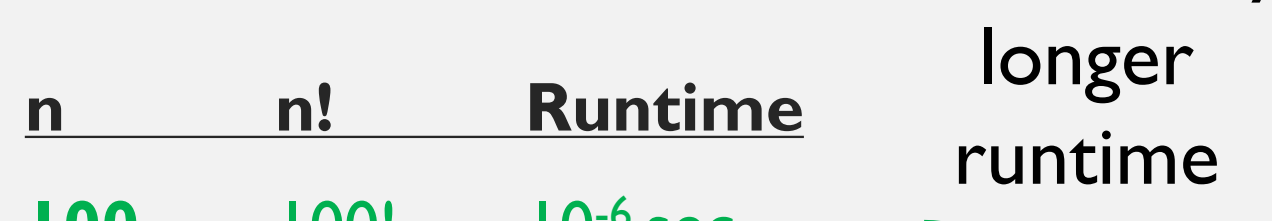

$102 \mathrm{I} 10 \mathrm{I} !>0.01 \mathrm{sec}$.

$104 \quad 102 !>1.8 \mathrm{~min}$.

$106 \quad 106 !>14$ days

108108 ! > $>45$ decades 


\section{BACKGROUND: NP-COMPLETENESS}

Computational complexity: Classify problems based on their inherent difficulty

Two possibilities

- P:

Set of problems that can be solved in polynomial time

- NP:

Set of problems whose solution can be verified in polynomial time

- NP-complete (NPC): The hardest problems in NP

- NP-hard (NPH): Problems at least as hard as the hardest problems in NP

- Known:

$P \subseteq N P$

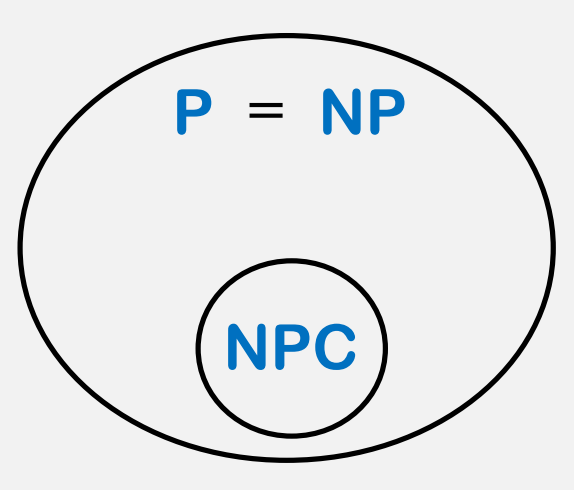

- Unknown:

$P \stackrel{?}{=} N P$

NP-complete
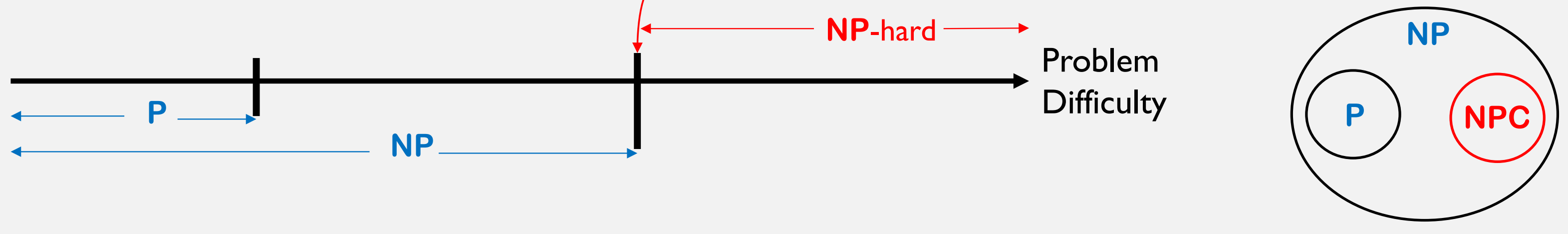


\section{ATM PROBLEM I: AIRSPACE SECTORIZATION PROBLEM (ASP)}

Airspace

Flight paths

Airport

Partition the airspace into sectors such that:

- Workload in each partition is $\leq c_{w}$.

- The number of sectors is $\leq c_{s}$

A set of flights

Maximum sector workload

Maximum sector count

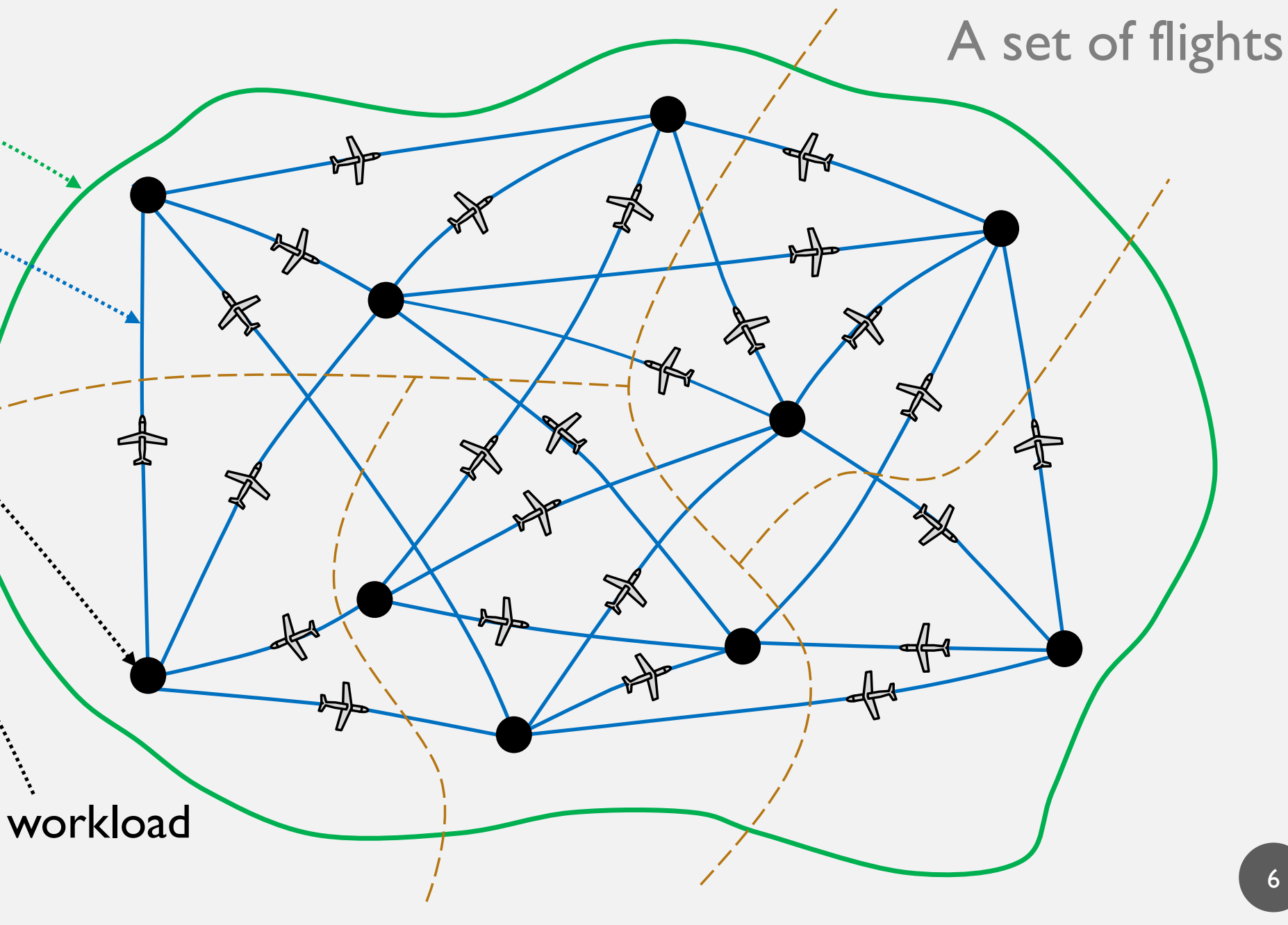




\section{ATM PROBLEM I: AIRSPACE SECTORIZATION PROBLEM (ASP)}

- Known problem: PLANAR-P3(6):

- Given a planar graph

- Each node connected to no more than 6 other nodes

- Question: Can we partition the nodes into sets of 3 , such that nodes in each set form a triangle)

- In this example the answer is YES

- Known to be NP-complete

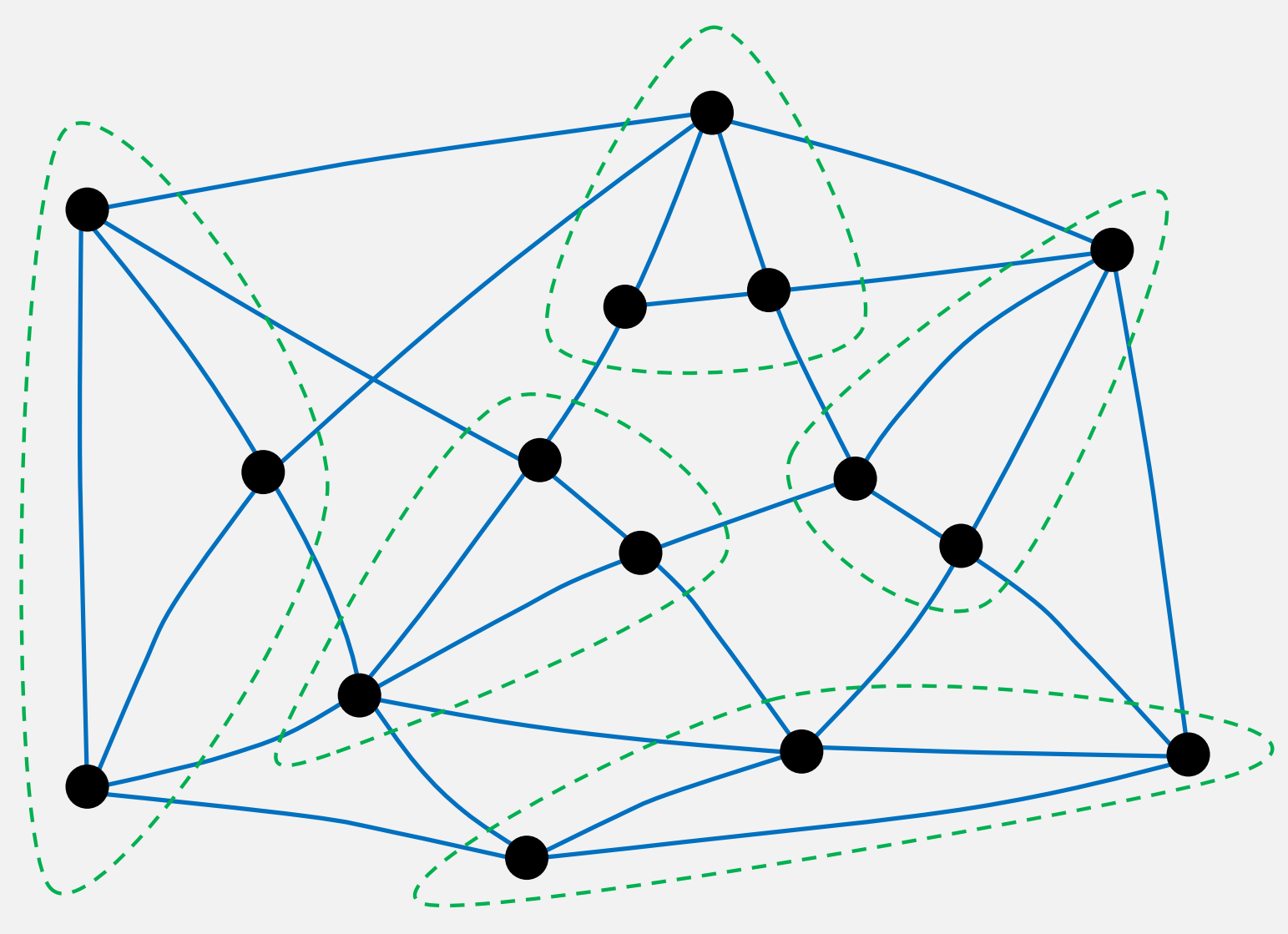




\section{ATM PROBLEM I: AIRSPACE SECTORIZATION PROBLEM (ASP)}

- It is known that ASP is NP-complete if sectors are required to be axis-aligned rectangles [Sabhnani, et al 2008]

- We transform PLANAR-P3(6) to ASP

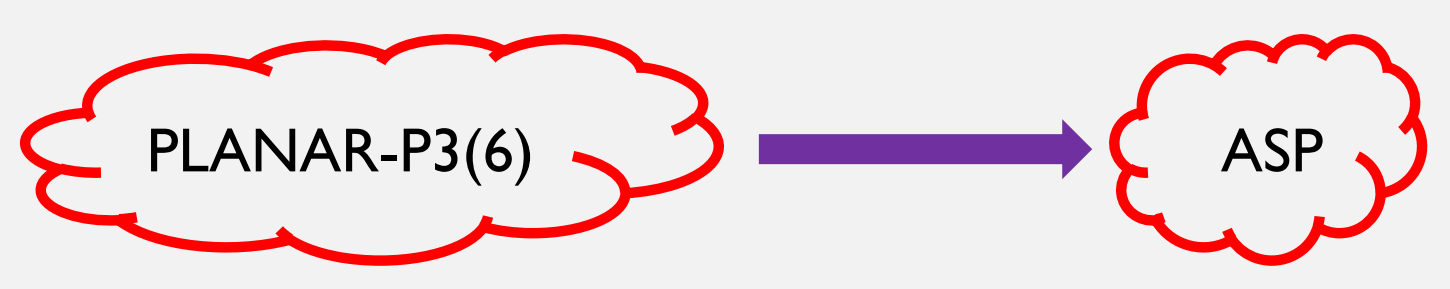

- Theorem I: ASP is NP-complete under several workload models, in general, even if the flight paths form a planar graph, and no more than 6 flights originate or terminate at each airport. 


\section{ATM PROBLEM 2:}

\section{MINIMUM DELAY SCHEDULING (MDS) IN TRAFFIC FLOW MANAGEMENT}

Airspace

Partitioned into sectors $s_{1}, s_{2}, s_{3}, \ldots$

Each sector $s_{i}$ has a capacity function $c_{i}(t)$

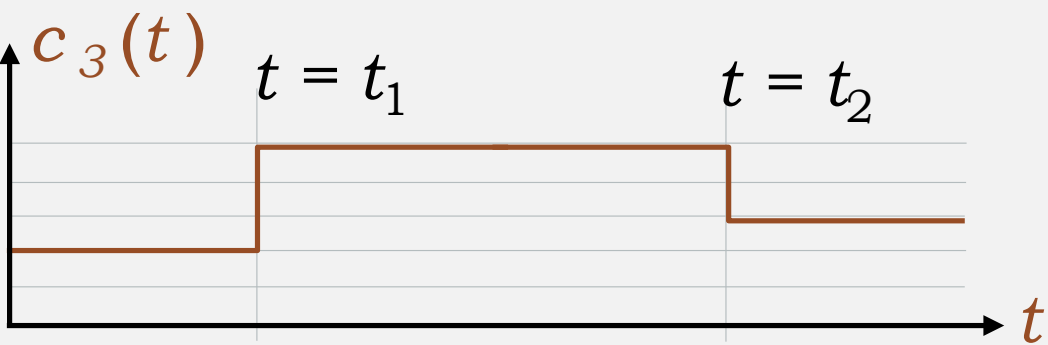

Each flight $f$ has a schedule

$\left[t_{0},\left(s_{1}, 4\right),\left(s_{2}, 6\right),\left(s_{3}, 5\right),\left(s_{7}, 3\right)\right]$

Assign delays to each flight on the ground or along its path to meet:

- Sector capacity constraints

- Airport arrival departure rate constraints (if any)

Objective: Minimize the sum of all delays imposed

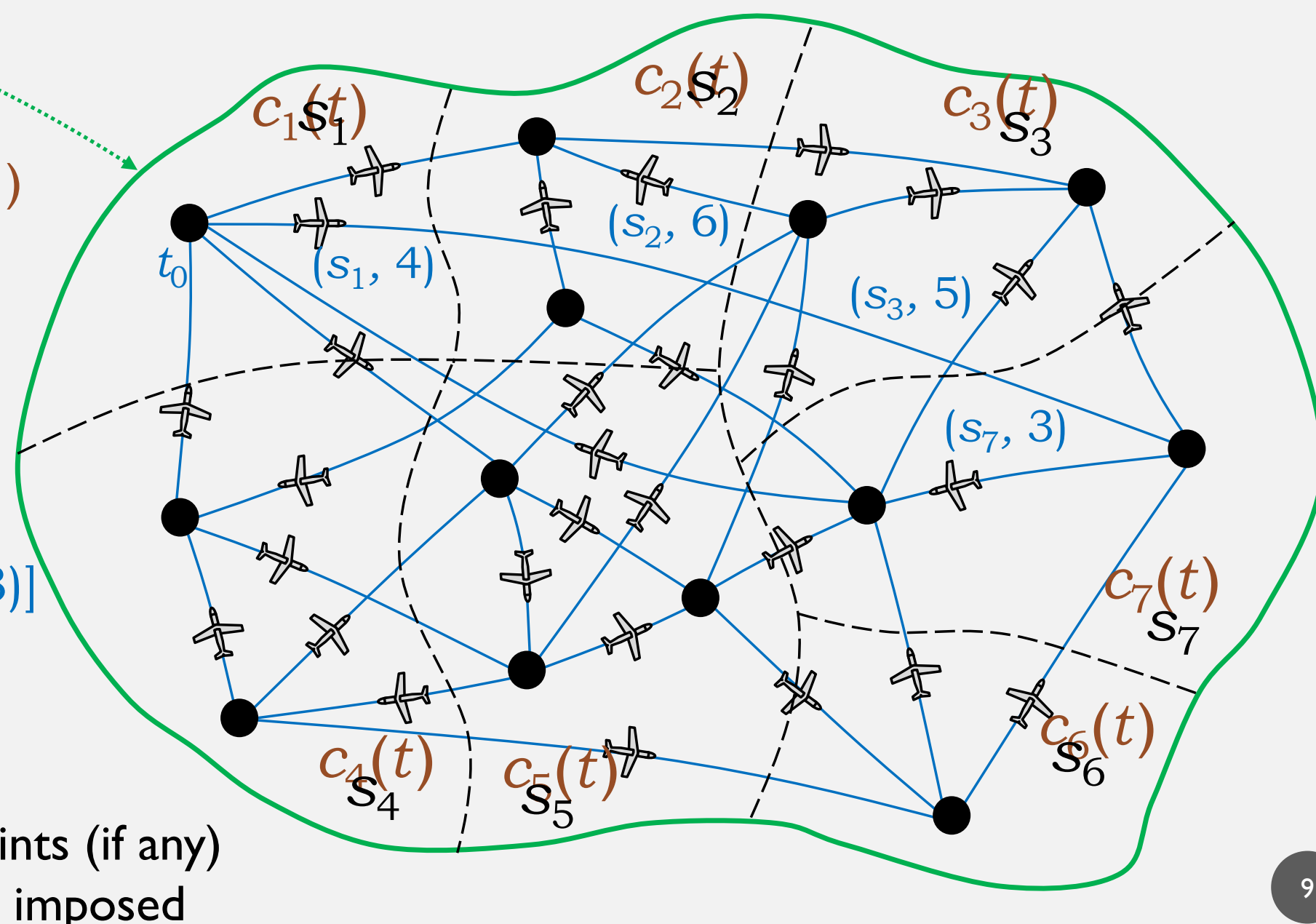


- Known problem: Maximum Independent Set (MIS) in graphs

- Given a graph

- Find its largest independent set

Subset of nodes, such that none of them is connected by an edge to any other node in the set

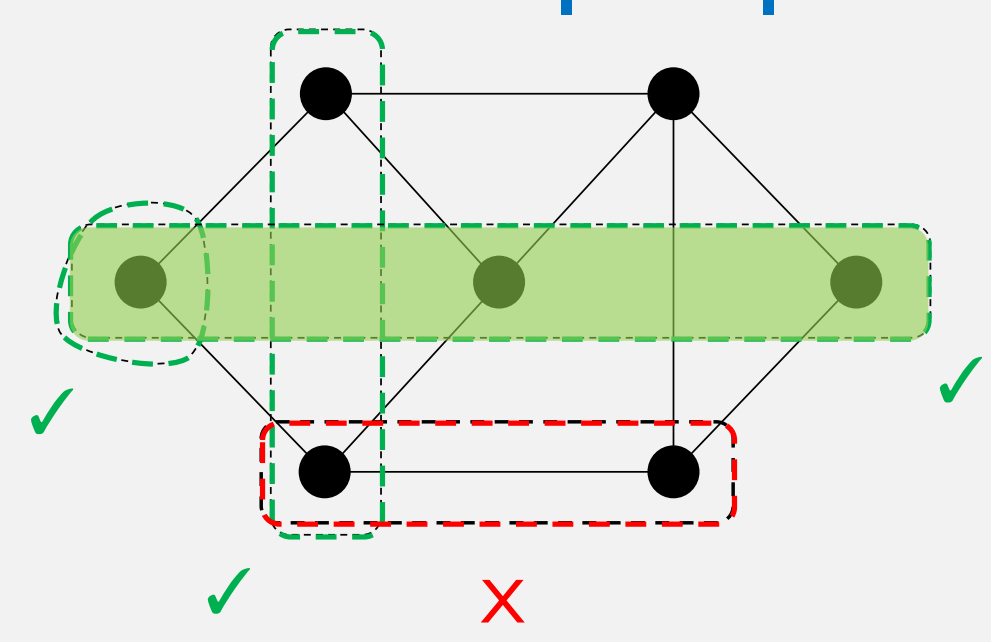

- Known to be NP-hard

- Known that unless $\mathbf{P}=\mathbf{N P}$, the problem cannot be approximated within polynomial time to within a factor $n^{1-\varepsilon}$ for any $\varepsilon>0$ 
- It is known that the MDS problem is NP-hard [Bertsimas et al 1998]

- We transform graph MIS problem to a simplified version of MDS problem
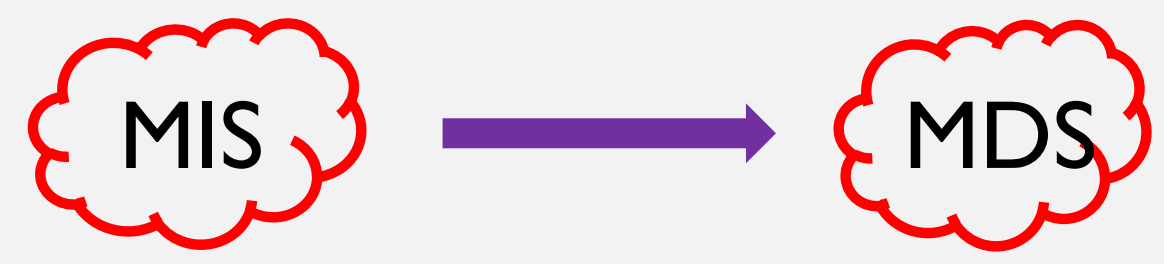

- Theorem 2: Unless $\mathbf{P}=$ NP, the MDS problem cannot be approximated in polynomial time to within a factor $n^{1-\varepsilon}$ for any $\varepsilon>0$, where $n$ is the number of aircraft in the problem instance, even if all the delays are to be taken on the ground prior to takeoff. 


\section{ATM PROBLEM 3:}

MAXIMUM SET OF DEPENDENT AIRCRAFT (MSDA) IN PRECISION ARRIVAL SCHEDULING

- Consider a set of aircraft $a, b, c, \ldots$

- Flying along their arrival routes following their prescribed schedules

- To land on their designated runways at the airport

- Due to off-nominal conditions, an aircraft may not meet its scheduled time slot and needs to be rescheduled

- We need to identify only the set of dependent aircraft who need to be rescheduled along with it

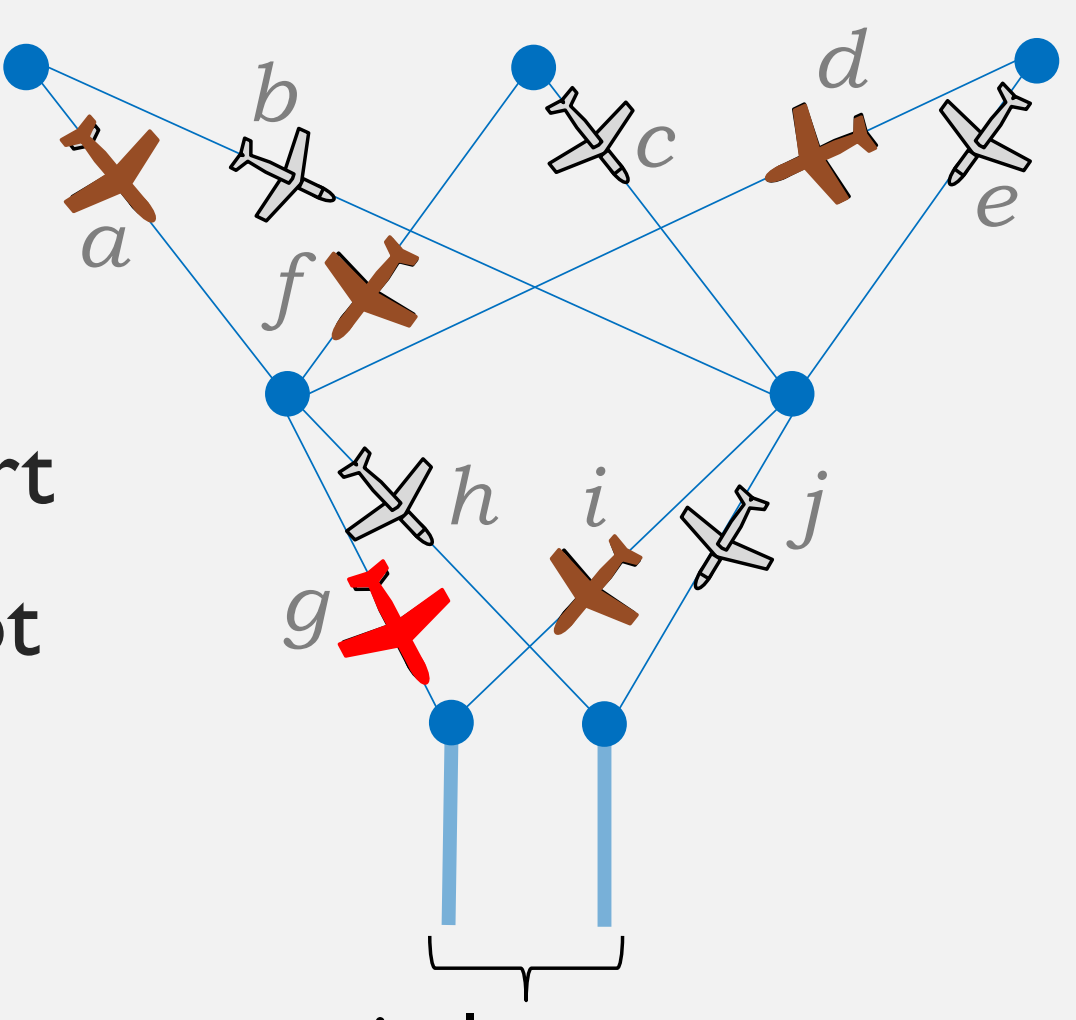

arrival runways 


\section{ATM PROBLEM 3:}

\section{MAXIMUM SET OF DEPENDENT AIRCRAFT (MSDA)}

IN PRECISION ARRIVAL SCHEDULING

- Known graph algorithm: Graph reachability (e.g. Breadth-First Search, Depth-First Search)

- Given a directed graph $G=(V, E)$ and a node $v \in V$ find the set of all nodes reachable from $v$

- Can be solved in $O(|V|+|E|)$ 


\section{ATM PROBLEM 3:}

\section{MAXIMUM SET OF DEPENDENT AIRCRAFT (MSDA) IN PRECISION ARRIVAL SCHEDULING}

- Transformed MSDA into graph reachability and solved it

- Monte Carlo Simulation

- 3 Airports: DAL, LAX, PHX

- 100 random scenarios

- 10, 20, 40, 80, 160 aircraft

- randomly chosen target

- Run-time $<0.5 \mathrm{sec}$.

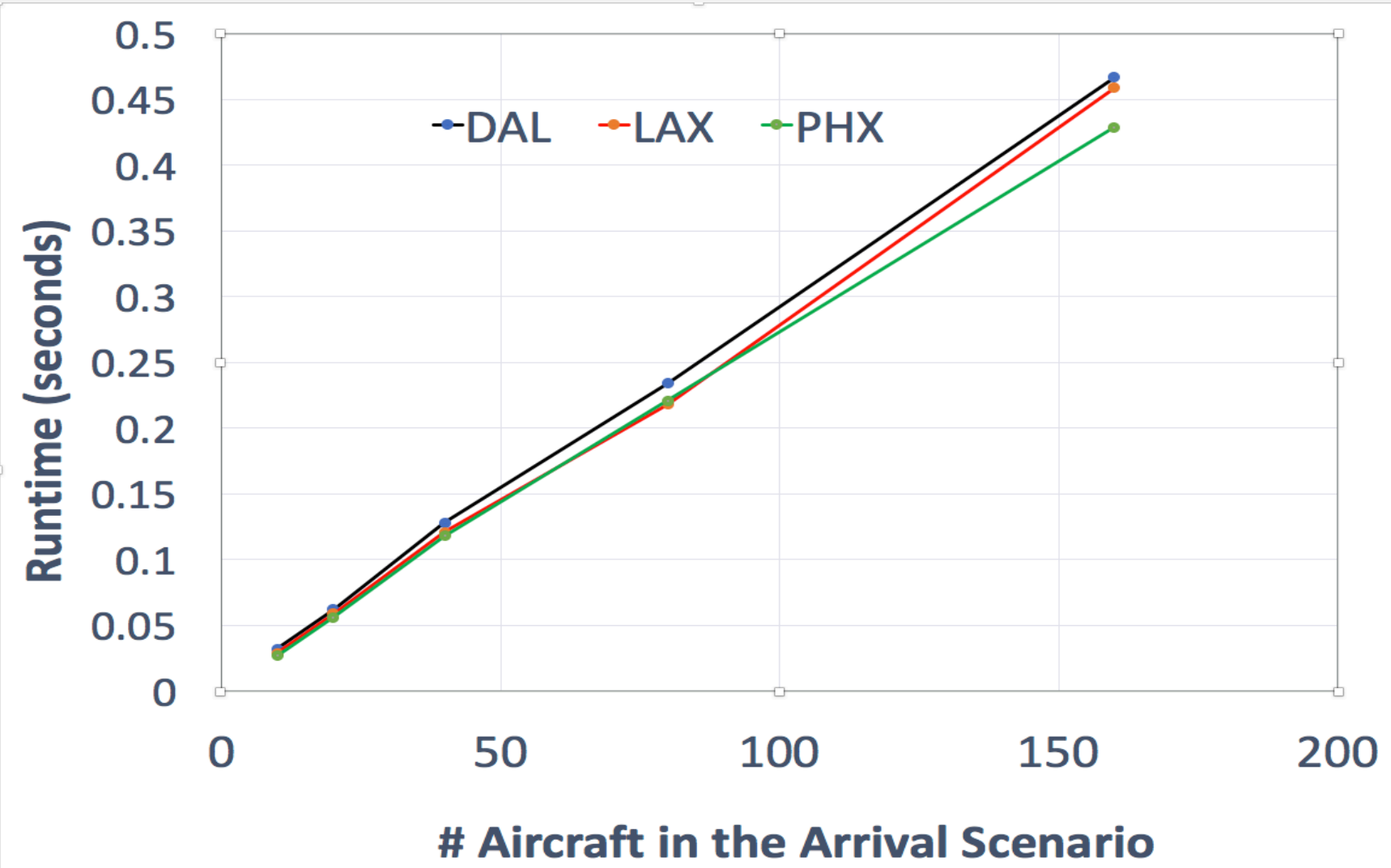




\section{SUMMARY}

- Studied three problems arising in ATM:

- Airspace Sectorization Problem (ASP): Showed it is NP-complete

- Min Delay Scheduling (MDS): Showed unless P = NP the problem cannot be approximated in polynomial time

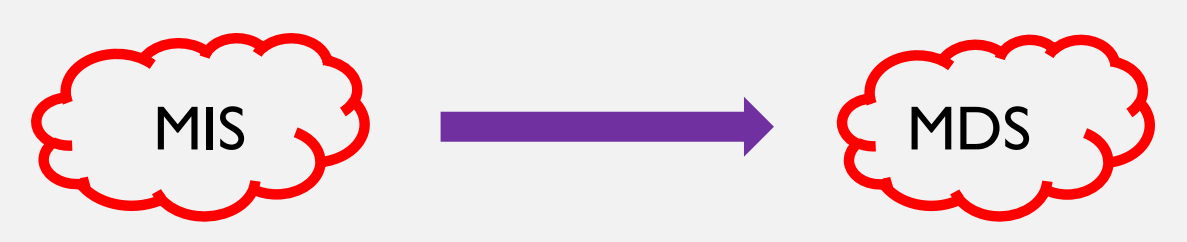

- Maximum Set of Dependent Aircraft (MSDA) in arrival scheduling: Solved using a very efficient algorithm

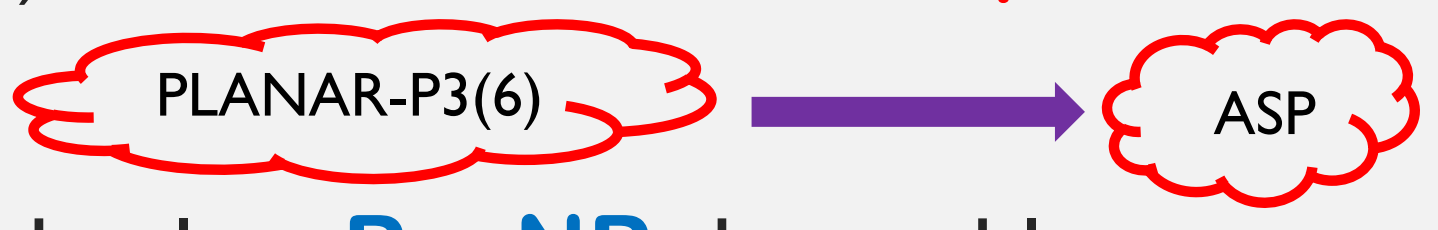




\section{CONCLUSION}

- Graph theory is a natural abstraction for many ATM problems

- Used known graph problems to learn about ATM problem

- Polynomial transformation can be used to

- Gain insights about inherent difficulty of new problems

- Solve new problems efficiently

- Linking problems allows:

- Reap the benefits of earlier or future development

- Fertilization across different technical disciplines 


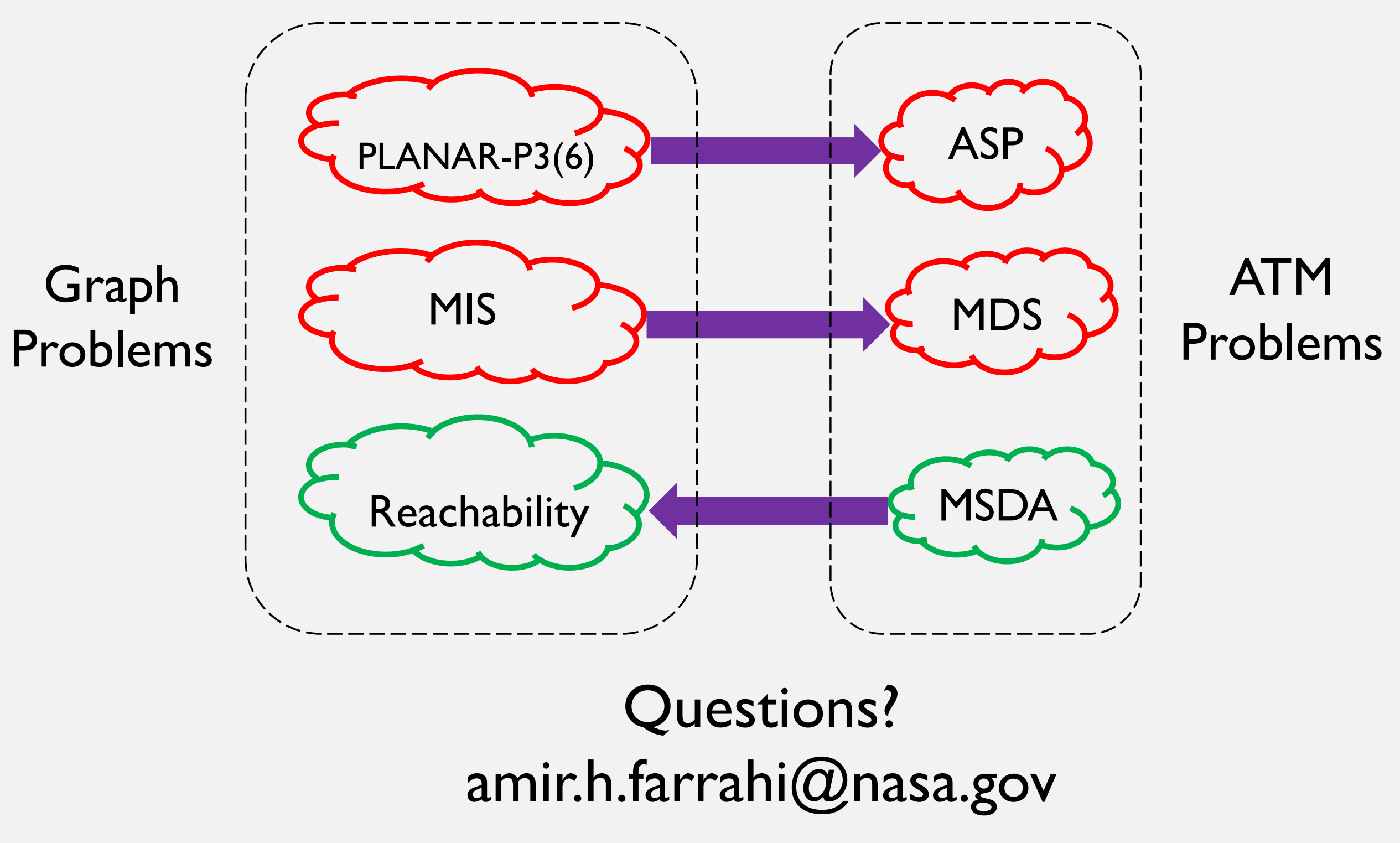




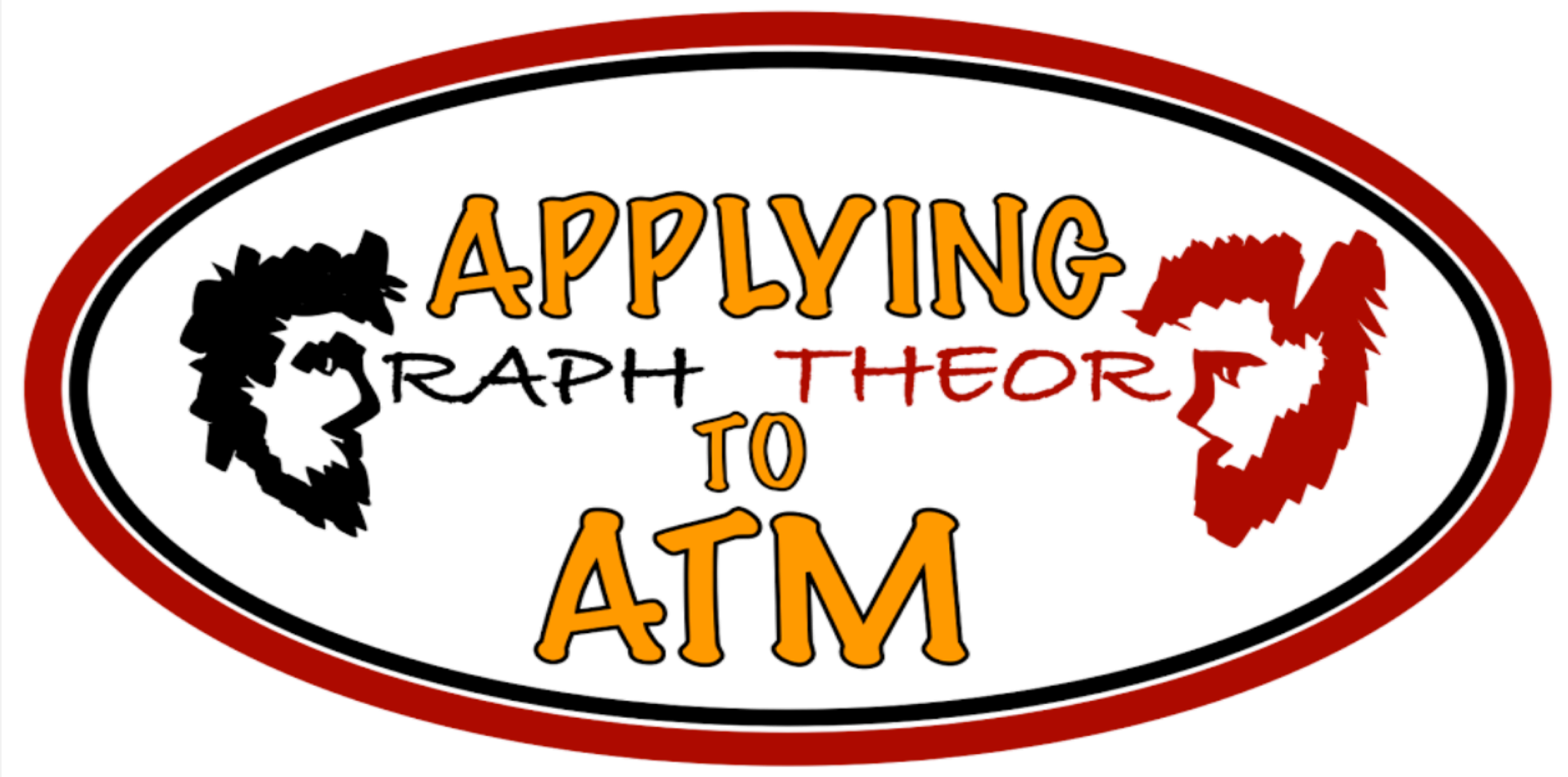

